\title{
Effects of radiation on growth of two human tumour cell lines surviving a previous high dose, low dose-rate, radionuclide exposure
}

\author{
LOVA SEGERSTRÖM, BO STENERLOW, VERONIKA ERIKSSON and JÖRGEN CARLSSON \\ Unit of Biomedical Radiation Sciences, Department of Oncology, Radiology and Clinical Immunology, \\ Rudbeck Laboratory, Uppsala University, SE-751 85 Uppsala, Sweden
}

Received February 20,2008; Accepted April 16, 2008

DOI: 10.3892/ijo_00000014

\begin{abstract}
Effects of radiation on growth of two human tumour cell lines that survived a previous high dose, low dose-rate radionuclide exposure simulating intensive radionuclide therapy, were analyzed. The purpose was to investigate whether the survivors gained therapy induced changes in growth and radiation response. The U118MG, ParRes (parental resistant), and U373MG, ParSen (parental sensitive), glioma cells were used because they are known to be low dose-rate radiation resistant and sensitive, respectively. These cells were initially exposed to high dose, low dose-rate radiation for $24 \mathrm{~h}$ and surviving U118MG and U373MG cells formed new cultures called SurRes (surviving resistant) and SurSen (surviving sensitive), respectively. All four cell types were then exposed to graded acute radiation doses, 0-8 Gy, and analyzed for radiation induced growth disturbances. They were also analyzed regarding DNA-content and cell cycle distributions. The SurRes cells regained in most cases the same growth rate, had the same growth delays and showed generally a similar response as the original ParRes cells to the 0-8 Gy exposures. In contrast, the SurSen cells had in all cases slower growth rate and longer growth delays than the original ParSen cells after the 0-8 Gy exposures. There were no signs of radiation-induced radioresistance. The slow growing SurSen cells contained about $80 \%$ more DNA and had more cells in $G_{1}$ and fewer in $G_{2}$ than the ParSen cells. The conclusion is that tumour cells surviving high dose, low dose-rate, radionuclide therapy, afterwards can react differently to a new radiation exposure.
\end{abstract}

\section{Introduction}

Radionuclide therapy is presently used for treatment of lymphomas $(1,2)$ using radiolabelled antibodies. It is also

Correspondence to: Professor Jörgen Carlsson, Division of Biomedical Radiation Sciences, Rudbeck Laboratory, Uppsala University, SE-751 85 Uppsala, Sweden

E-mail: jorgen.carlsson@bms.uu.se

Key words: cancer, cultured cells, glioma, growth, low dose-rate, radionuclides, radiotherapy used for neuroendocrine $(3,4)$ and paediatric tumours (5) using radiolabelled somatostatin analogues and $\mathrm{mIBG}$, respectively. In the majority of those cases beta emitters such as ${ }^{90} \mathrm{Y},{ }^{131} \mathrm{I}$ or ${ }^{177} \mathrm{Lu}$ have been applied. The results have, so far, essentially shown palliative effects $(2,6-8)$ and there is hope that combinations of different beta particle emitters, e.g. ${ }^{90} \mathrm{Y}$ and ${ }^{177} \mathrm{Lu}$, will further improve the therapy results (9). The introduction of radionuclide therapy for gliomas is an interesting option and has only been tried in a limited number of cases (10).

Radionuclide therapy is characterized by the delivery of rather high radiation doses but given with low dose-rate. The dose-rates in targeted low-LET (linear energy transfer) radionuclide therapy are typically in the order of $0.01-1.0 \mathrm{~Gy} / \mathrm{h}$ $(6,11-14)$ which is much lower than the $0.5-2.0 \mathrm{~Gy} / \mathrm{min}$ used in conventional external radiotherapy $(15,16)$. Low dose-rate allows for DNA repair and cellular repopulation during the radiation exposure, which is not the case during high doserate exposures $(16,17)$.

The idea behind this study was that low dose-rate irradiated tumour cells that survived a targeted low-LET radionuclide therapy, in spite of the high total radiation dose, were expanded to new cell cultures. The question was then whether the survivors have changed their growth properties as seen after new radiation exposures. It is, of course, not possible to give a general answer by studying only two tumour cell lines, one known to be resistant to low dose-rate radiation and one known to be sensitive to such exposure. However, the studies can at least give two examples of what can happen. Actually, there are reasons to believe that tumour cells might have changed properties after therapy. Genomic instability with its elevated mutation rates together with the selective pressure of radiation therapy can, at least in theory, be a ground for the development of sub-clones with changed growth properties and these cells could possibly contribute to therapy failure (18).

In this study, all radiation exposures were given to samples containing (0.5-1.0) x $10^{5}$ tumour cells. The choice of about $10^{5}$ cells was somewhat arbitrary and is based on arguments previously described (19). This number represents a small tumour cell cluster that cannot be identified by diagnostic routine procedures such as CT or MRI unless the tumour cells cause macroscopic changes in the surrounding normal tissues. Furthermore, this number of tumour cells does in 
most cases not give symptoms for the patient. Thus, a cluster of about $10^{5}$ tumour cells can be considered a subclinical tumour. It should also be noted that about $10^{5}$ tumour cells in a normal cell culture dish or flask give enough space to allow for exponential growth and, at the same time, frequent cell-cell contacts.

To kill about $10^{5}$ cells with radiation, it is necessary to reach a survival probability for each cell of $<10^{-5}$. It is uncertain which radiation dose must be applied for that since the experimental data in conventional survival curves is not valid for low survival levels and high radiation doses. The shape of survival curves is also uncertain when the radiation is given at low dose-rate. However, a recent study on effects of low dose-rates on cellular long-term growth has indicated that total radiation doses in the range 20-40 Gy, given with doserates $0.1-1.0 \mathrm{~Gy} / \mathrm{h}$, are necessary to have a reasonable chance to kill about $10^{5}$ cells. The doses around 20 Gy are for sensitive cells and about 40 Gy for resistant cells (19).

There are several factors that determine the dose-rate in radionuclide therapy of solid tumours and locally spread tumour cells, such as injected amount of radioactivity and variations in vessel wall leakage and changes in blood flow. In addition, there might be time-dependent variations in the expression of target structures on the tumour cells. The achievable dose-rates are to a large extent also a consequence of the cross-fire effect. This means that radionuclides associated to one cell also irradiate neighbouring cells due to the long range of the radiation $(20,21)$ and can increase the dose-rate 10 -fold. It is therefore reasonable that dose-rates in the range we have used in the initial selection experiments in this study can be achieved in a patient tumour, i.e. $\leq 1 \mathrm{~Gy} / \mathrm{h}$. Futhermore, beta particles with long range will enable rather uniform dose-distributions and hopefully give therapeutic relevant radiation doses also to non-targeted tumour cells.

Lower dose-rates than $0.1 \mathrm{~Gy} / \mathrm{h}$ will probably not lead to curative treatments when beta particles are applied to kill clusters containing about $10^{5}$ cells (19). The dose-rate will actually be $<0.1 \mathrm{~Gy} / \mathrm{h}$ for a single isolated tumour cell (no cross fire effect) considering only the radionuclides bound to that cell (22), which is an obvious drawback, but the dose might anyhow be enough to kill that single cell.

In this study, effects of radiation on growth of two tumour cell lines that survived a previous high dose, low dose-rate radiation exposure, simulating intensive radionuclide therapy was analyzed. The purpose was to investigate whether the survivors had gained therapy induced changes that could be seen in the response to new radiation exposures.

\section{Materials and methods}

Cells. The parental cells used were the two gliomas U118MG and U373MG, called ParRes (parental resistant) amd ParSen (parental sensitive), respectively. The cells were obtained from the Department of Genetics and Pathology, Uppsala University, Uppsala, Sweden, and were selected because they are low dose-rate radiation resistant and sensitive, respectively (19). Both cell types have recently been used in studies on effects of low dose-rate irradiations $(19,23)$. The cells were grown in Ham's F-10 medium supplemented with $10 \%$ fetal calf serum, $2 \mathrm{mmol} / \mathrm{l} \mathrm{L-glutamine,} 100 \mu \mathrm{g} / \mathrm{ml}$ streptomycin and $100 \mathrm{U} / \mathrm{ml}$ penicillin, all components purchased from Biological Industries (Beit Haemek, Israel) or from Biochrom AG (Berlin, Germany). The cells were normally grown in an incubator of type Galaxy S (LabRum Klimat AB, Stockholm, Sweden) and during the experiments they were grown in a similar incubator (Kebo Assab T304GF, Stockholm, Sweden). Both incubators were run at $37^{\circ} \mathrm{C}$ and contained $5 \%$ carbon dioxide in a humidified atmosphere.

Cell counting. The medium was removed from the cell dishes and the cells were quickly washed with $0.5 \mathrm{ml}$ trypsin-EDTA ( $0.25 \%$ trypsin/ $0.02 \%$ EDTA solution in PBS, Merck Eurolab, Stockholm, Sweden) and then incubated with $0.5 \mathrm{ml}$ trypsinEDTA $\left(37^{\circ} \mathrm{C}, 5 \% \mathrm{CO}_{2}\right)$ until the cells detached. Next, $1.5 \mathrm{ml}$ medium was added to each dish and the cells resuspended to a single cell solution. For counting, $19.5 \mathrm{ml} 1 \mathrm{X}$ PBS (pH 7.4) was added to $0.5 \mathrm{ml}$ cell suspension and an electronic cell counter was used (Coulter Z2, 7-20 $\mu \mathrm{m}$, Beckman Coulter, Stockholm, Sweden).

Growth curves and growth delays. Growth curves were constructed as if all cells would have been saved at each subcultivation. By calculating how many cells that would have been obtained if all cells were saved, such high cell numbers as e.g. $10^{14}$ and higher were obtained. In reality, repeated dilutions were made to keep the number of cells in each culture flask in the range of $10^{5}-10^{6}$, thus allowing for exponential growth. Four parallel flasks were kept for each experiment. Doubling times were determined through analysis of the slope of the growth curves during exponential growth. Growth delay was defined as the time difference, measured as the number of days, between the irradiated cells and the control cells to reach a cell number of $10^{10}$.

Low dose-rate radiation chambers. The irradiation chambers for low dose-rate beta irradiations and the dose-rate calibrations have previously been described (23). The irradiation chambers were loaded by adding the beta emitter ${ }^{32} \mathrm{P}$ (Orthophosphate, Amersham Pharmacia Biotech, Amersham, UK), with the half-life 14.3 days. Six $\mathrm{ml}{ }^{32} \mathrm{P}$ solution, at loading containing $\sim 2.22 \mathrm{GBq}$, was dissolved in $400 \mathrm{ml}$ water. The dose-rate was controlled by measurements with a thin-walled ion chamber (23). The beta irradiated cells were grown in 3-cm diameter culture dishes with a plastic bottom thickness of $1 \mathrm{~mm}$ above the ${ }^{32} \mathrm{P}$ source. The chambers were at $37^{\circ} \mathrm{C}$ in cell culture incubators (Kebo Assab T304GF, Stockholm, Sweden) containing $5 \%$ carbon dioxide.

Sensitivity for low dose-rate exposures. The sensitivity for low dose-rate radiation of both U118MG and U373MG cells has been characterized in a recently published study from which the results given in Fig. 1 are taken (19). It is seen that radiation doses in the order of $40 \mathrm{~Gy}$ are necessary for killing of $10^{5} \mathrm{U} 118 \mathrm{MG}$ cells while only about $20 \mathrm{~Gy}$ seems to be necessary to kill $10^{5} \mathrm{U} 373 \mathrm{MG}$ cells with low dose-rate radiation.

The results in Fig. 1 show that the U118MG cells were more resistant than the U373MG cells to the low dose-rate treatments. The shift from recovery to 'cure' fell within a rather narrow range of dose-rate and exposure time combi- 

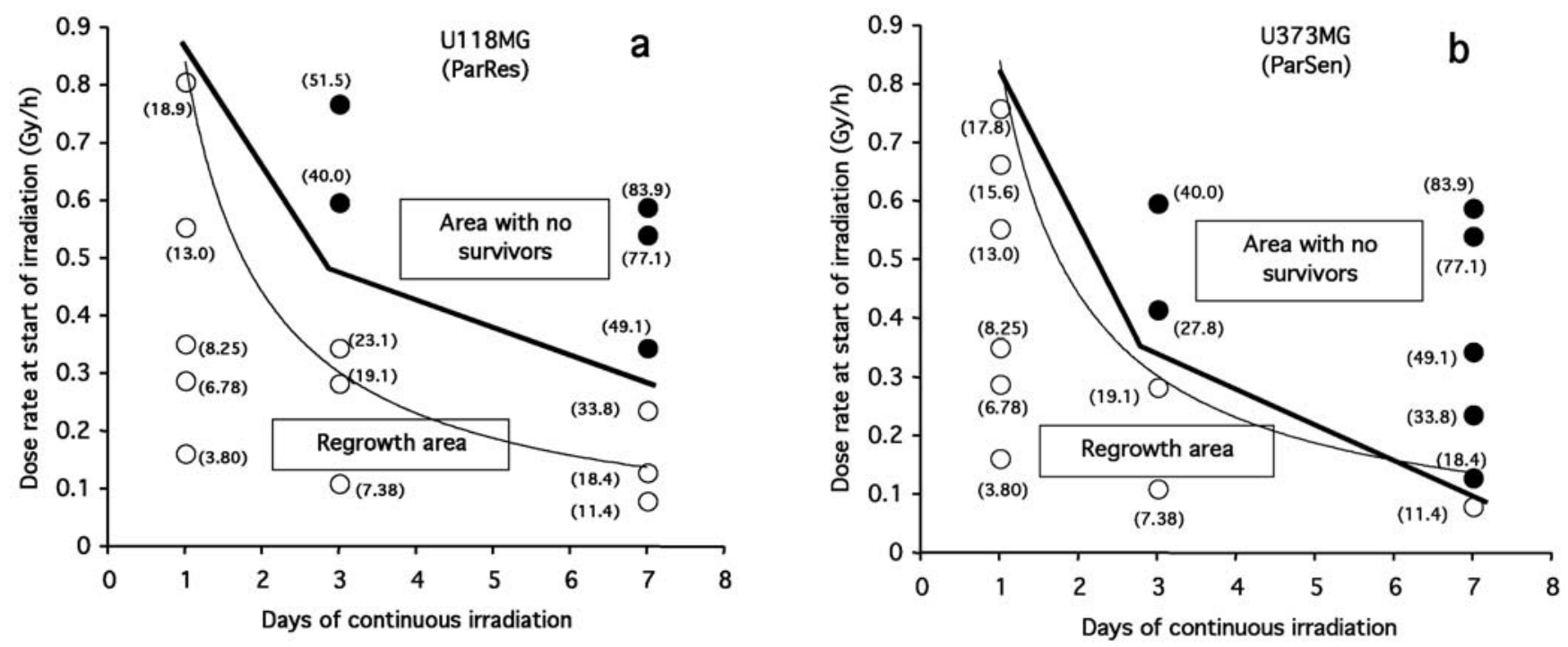

Figure 1. Summary of low dose-rate experiments carried out for therapy of 105 U118MG (ParRes) (a) and U373MG (ParSen) (b) glioma cells. The cells were irradiated with different initial dose-rates and were then exposed to the radiation for 1, 3 or 7 days. The figures show at which combinations of dose-rate and exposure time all cells were killed (area with no survivors, filled circles), and at which at least some cells survived and gave regrowth (the regrowth area, open circles). The separation between the two areas is indicated with bold solid lines. The total delivered radiation dose (Gy) is given in the parenthesis near each point. The 20 Gy isodose curve is indicated with a dashed line. Reproduced from Carlsson et al (19) with kind permission of Springer Science and Business Media.

nations. The highest studied dose-rate, $\sim 0.8 \mathrm{~Gy} / \mathrm{h}$, is probably near the highest values that can be achieved in targeted radionuclide therapy $(11,12,19)$. The total radiation doses achieved after 1, 3 or 7 days exposure correspond to achievable doses in targeted radionuclide therapy (11), but most often total doses of $\leq 10-20$ Gy are obtained in such treatments of B-cell lymphomas (13). The U118MG cells are known to have hyperradiosensitivity, HRS (i.e. high radiosensitivity at doses $<0.5 \mathrm{~Gy}$ ) while this is not the case for the U373MG cells (24). However, HRS seems not to be of importance when these two types of cells are irradiated with low dose-rate $(19,23)$.

Cell culture conditions during and after the low dose-rate irradiations. The parental cells were seeded sparsely in culture dishes (diameter $3.5 \mathrm{~cm}$, surface $9.6 \mathrm{~cm}^{2}$, Nunc, Roskilde, Denmark) a few days before the start of the low dose-rate irradiations. They were seeded so that each culture dish contained about $10^{5}$ cells at the start of the radiation exposure. The U118MG and U373MG cells were irradiated for $24 \mathrm{~h}$ with beta particles from ${ }^{32} \mathrm{P}$ at low dose-rates, $\sim 0.81$ and $0.66 \mathrm{~Gy} / \mathrm{h}$, up to doses of $\sim 19.0$ and $15.5 \mathrm{~Gy}$, respectively. The irradiated cells were grown on the ${ }^{32} \mathrm{P}$-chamber and nonirradiated control dishes were placed at a radiation shielded area in the same incubator. The cells did not reach confluence during the irradiation period. After the radiation exposure, the cells were moved from the irradiation chamber incubator to another incubator and the cell growth was followed for several months. At the first subcultivation after the radiation exposure, the cells were transferred from culture dishes to culture flasks $\left(25 \mathrm{~cm}^{2}\right.$, Nunc), and such flasks were then used throughout the whole growth period. The culture medium was replaced three times a week. Cell counting was in most cases performed once a week followed by reseeding of $10^{5}$ cells in each new flask.
Handling of the survivors from the low dose-rate irradiations. The parental U118MG cells, called ParRes, and the U373MG cells, called ParSen, surviving the low dose-rate irradiations were taken at indicated times (see Results) and were expanded to new monolayer cultures. The surviving ParRes and ParSen cells were from now and on called SurRes (surviving resistant) and SurSen (surviving sensitive), respectively. The survivors were grown under standard culture conditions for two weeks as new monolayer cultures before continued experiments applying high dose-rate photon exposures as described below.

Photon irradiations. Acute photon irradiations in the dose range 0-8 Gy were carried out to analyze if there were any differences in the radiosensitivity between the parental ParRes and ParSen cells and the survivors SurRes and SurSen. All dishes, including controls, were transferred to the irradiation source $\left({ }^{137} \mathrm{Cs}\right.$ Gammacell 40 Exactor, MDS Nordion, Nuklex, Uppsala, Sweden) in a plastic box with a carbon filter. Cells in exponential growth were exposed to $0,2,4,6$ or 8 Gy, with an acute dose-rate of $1.40 \mathrm{~Gy} / \mathrm{min}$. The cells were then analyzed for growth, growth delays and radiosensitivity as described above. Approximately $0.5 \times 10^{5}$ trypsinated cells from each radiation dose were seeded. The cells were then grown until nearly confluent, trypsinated, counted and $0.5 \times 10^{5}$ cells seeded again. This was repeated several times and growth curves were obtained the same way as described in Growth curves and growth delays.

Growth extrapolation assay. Cells that do not grow sparsely and/or can not form distinct colonies are not suitable for the clonogenic survival assay. An alternative method is the growth extrapolation assay to determine 'intrinsic' radiosensitivity. It has been confirmed that the clonogenic survival assay and 
the growth curve assay can give comparable results (25-27). The principal difference between these two approaches is that the clonogenic survival assay measures the reproductive capacity of individual cells in a rather short time interval (normally 1-2 weeks) whilst the growth extrapolation assay measures the reproductive capacity of an entire cell population over a longer time. The growth extrapolation assay was applied in this study since the glioma cells could not grow well as isolated cells as is necessary for the clonogenic survival assay. The growth curves were generated as described above and when all the cultures had reached exponential growth, the exponential curve was extrapolated back to the time of exposure, to determine the number of cells that seemed to be responsible for the regrowth. From this, an approximate estimate of the surviving fraction could be calculated.

Cell cycle analysis. It was of interest to analyze possible changes between the parental ParRes and ParSen and the surviving SurRes and SurSen cells. The cell cycle analysis method has previously been described (23) and is only briefly described here. Two hours before cell harvest, BrdU (Sigma, St. Louis, MO, USA) was added to a final concentration $10^{-4} \mathrm{M}$. After cell harvest, the cells were washed, fixed in $70 \%$ ethanol, and stored at $4^{\circ} \mathrm{C}$ in the dark until staining and analysis. The fixed cells were centrifuged, ethanol was removed and about $10^{6}$ cells were suspended in $2 \mathrm{ml} 4 \mathrm{M} \mathrm{HCl}$. After $20 \mathrm{~min}, 5 \mathrm{ml}$ of PBS was added, the tubes were vortexed and centrifuged for $5 \mathrm{~min}$ at $600 \mathrm{x} \mathrm{g}$. This procedure was repeated twice using wash solution ( $2 \%$ bovine serum albumin in PBS). After the last wash the cell pellet was vortexed and $10 \mu \mathrm{l}$ of an FITC-tagged anti-BrdU antibody (F7210, Dako, Glostrup, Denmark) was added. After $30 \mathrm{~min}$ of incubation at $4^{\circ} \mathrm{C}$, the cells were washed two times with wash solution as above to remove unbound antibody. After the last wash, $0.5 \mathrm{ml}$ PBS with $100 \mu \mathrm{g} / \mathrm{ml}$ propidium iodide (PI; Sigma, St Louis, MO, USA) was added during vortex mixing, thereafter the samples were left at $4^{\circ} \mathrm{C}$ for at least $30 \mathrm{~min}$. The flowcytometric analysis was done using a FACSort (BD Biosciences, San Jose, CA, USA) flow cytometer. Forward scatter, side scatter, Fl1 (FITC) and Fl3 (PI) fluorescence signals were collected. The doublet discrimination module was used for the PI signals to restrict the analysis to single cells. Generally 30,000 events were collected from each sample. Data analysis and plotting were done using CellQuest software (BD Biosciences). Areas of interest for $G_{1}, S$ and $G_{2}$ phase cells were defined and the proportion of cells in each of these regions were used for the calculation of cell cycle distributions (23).

Determination of DNA content. For the determination of the amount of DNA, cells were treated as in the cell cycle analysis protocol but with some changes. After the BrdU-pulse the cells were fixated together with chicken red blood cells, CRBC, as a DNA standard, using $\sim 10^{4}$ chicken cells per $10^{6}$ tumour cells. The chicken cells were carried along with the tumour cells through the whole staining procedure. The only change in the staining protocol was that since the CRBC are smaller than the tumour cells all centrifugation times had to be doubled. In the FACS analysis information about both the cell cycle distribution and the DNA content could be gathered simul- taneously. The CRBC DNA content is $\sim 35 \%$ of the DNA content of a normal human diploid cells, so the DNA content could also be compared to diploid cells. Results are presented as the change in DNA content when compared to diploid cells and also when compared relative to each other.

Analysis of radiation-induced apoptosis. The apoptosis analysis method has previously been described (23) so only a short description is given here. The cells were in this study analyzed to assess their tendency to undergo apoptosis after an acute challenging high dose-rate exposure to gamma radiation. Apoptotic cells were found with the Annexin V-FACS analysis. Apoptotic cells can be distinguished by both morphological and biochemical changes. Apoptotic cells expose phosphatidylserine (PS) on the extra-cellular surface of the cytoplasmic membrane, whereas in healthy cells PS is located on the inside of the membrane. The translocation of PS to the outer side of the membrane on apoptotic cells targets them for phagocytosis of macrophages. Annexin V is a human vascular anticoagulant, a $\mathrm{Ca}^{2+}$-dependent phospholipids binding protein that binds with high affinity to PS. Annexin V is conjugated to a fluorescent dye that is detected by FACS. In dead or fragmented cells, Annexin V can enter the membrane and binds to PS on the inside of the cells thus giving a false-positive result. This can be circumvented by counterstaining the dead cells with propidium iodine that is only taken up by dead cells, so that all double positive (i.e. both Annexin $\mathrm{V}$ and propidium iodine positive) cells are excluded from the results. Cells were grown to half confluence and gamma irradiated with the doses, 0 (control), 5 and 9 Gy. Immediately prior to radiation the media of the cells were changed, and after radiation the cells were incubated for three days in the same media. The 3-day incubations and the radiation dose levels were chosen based on two articles stating that radiation-induced apoptosis detected with the Annexin V assay peaked after about three days for other analyzed cell lines in the dose interval from 2-10 Gy $(28,29)$. Both detached and attached cells, three days after radiation exposure, were analyzed. The detached cells were counted and centrifuged at $1200 \mathrm{rpm}$ $(400 \mathrm{x} \mathrm{g})$ for $10 \mathrm{~min}$. The attached cells were trypsinated, counted and centrifuged the same way. All cell pellets were then washed in PBS and suspended in cold Annexin binding buffer (10 mM HEPES, $140 \mathrm{mM} \mathrm{NaCl}, 2,5 \mathrm{mM} \mathrm{CaCl}_{2}, \mathrm{pH}$ 7.4) to a concentration of $10^{6}$ cells $/ \mathrm{ml}$ buffer and kept on ice. Cell/buffer solution $(100 \mu \mathrm{l})$ was used for each assay, mixed with $5 \mu 1$ Annexin V conjugated with Alexa Fluor 488 (Molecular Probes, Eugene, Oregon, USA) and incubated for $15 \mathrm{~min}$ in RT. Then $400 \mu \mathrm{l}$ of buffer together with $5 \mu 1$ $(10 \mu \mathrm{g} / \mathrm{ml})$ propidium iodine was added and the solution was kept on ice until analysis on the FACSort. Areas of interest were defined using the CellQuest software and the amount of apoptotic cells was presented as percentages with standard deviations (23).

\section{Results}

Survival of the low dose-rate high dose beta irradiated cells. Fig. 2 shows growth curves for the parental U118MG cells, called ParRes (Fig. 2a) and parental U373MG cells, called ParSen (Fig. 2b), irradiated for $24 \mathrm{~h}$ with beta particles at low 

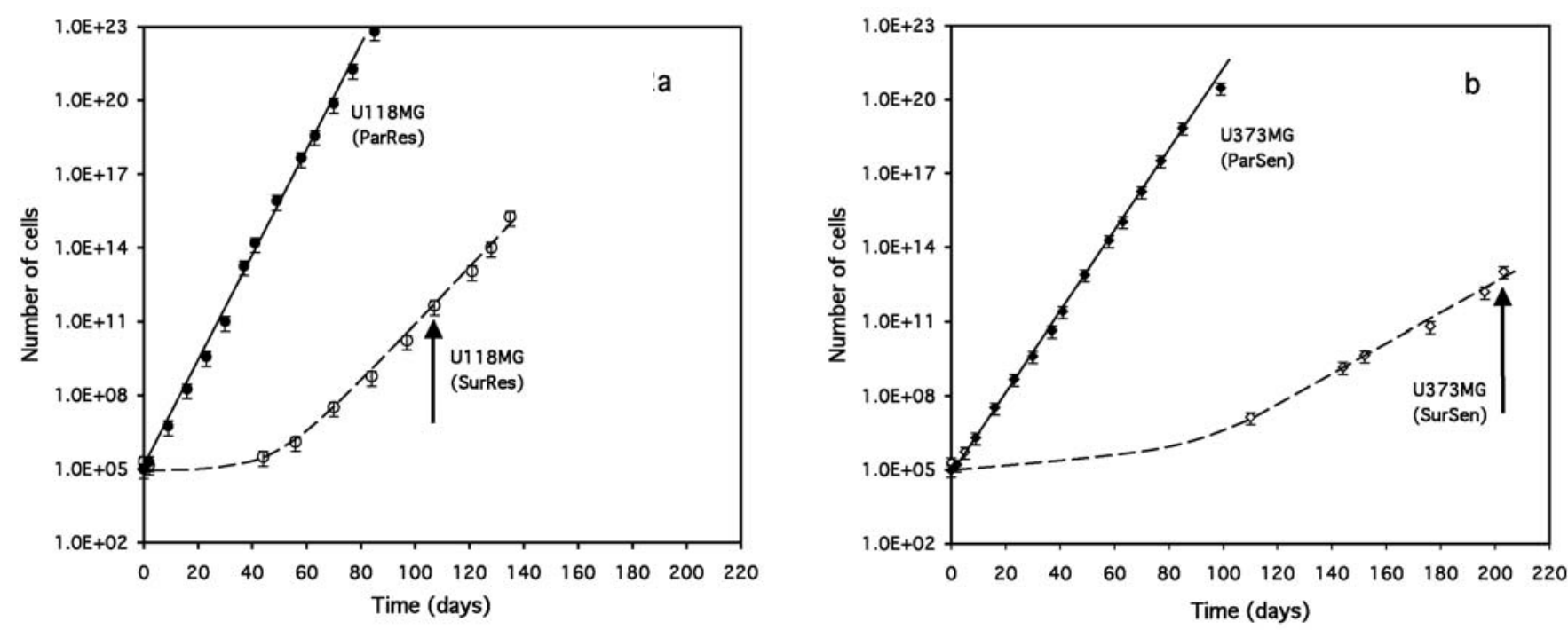

Figure 2. Cell growth as a function of time after exposure to low dose-rate irradiation. The U118MG (ParRes) cells (a) were exposed to 0.81 Gy/h as the initial dose-rate and then continuously exposed for $24 \mathrm{~h}$ (total dose $19.0 \mathrm{~Gy}$ ). The U373MG (ParSen) cells (b) were exposed to $0.66 \mathrm{~Gy} / \mathrm{h}$ as the initial dose-rate and then continuously exposed for $24 \mathrm{~h}$ (total dose $15.5 \mathrm{~Gy}$ ). The values for non-irradiated control cells are shown with filled symbols and solid lines and the values for low dose-rate irradiated cells with open symbols and dashed lines. Surviving cells were taken at the times indicated with arrows and grew as monolayer cultures called the SurRes (a) and SurSen (b) cells. Mean values and maximal variations from four parallel samples.

dose-rates up to doses of about 19.0 and $15.5 \mathrm{~Gy}$, respectively. The surviving U118MG and U373MG cells were, after a follow-up time of 4 and 6.5 months, respectively, collected called SurRes (U118MG-surviving resistant) (Fig. 2a) and SurSen (U373MG-surviving sensitive) (Fig. 2b). The arrows in Fig. 2 indicate the times for harvest of the surviving cells that then were used in the experiments described below.

The ParRes cells grew as bipolar fibroblast-like cells. The surviving SurRes cells maintained the morphology of the parental cells but with some minor changes. The SurRes cells tended to be somewhat smaller and have slightly shorter cytoplasmic extensions. The colony forming ability when grown as monolayer was low for both ParRes and SurRes cells. They also tended to migrate fast and did not form distinct colonies.

The ParSen cells were pleomorphic and grew as islands containing a dense mosaic cell-cell pattern. The SurSen cells had, in comparison to the ParSen cells, a decreased growth rate and also changes in cell morphology, such as larger cytoplasm and nucleus. Both the ParSen and SurSen cells had, when separated to isolated single cells, low cloning capacity.

Growth curves after acute 0-8 Gy exposures. Growth curves for all four cell types after high dose-rate exposures to $0,2,4$, 6 or 8 Gy are shown in Fig. 3. The slope of the growth curves for non-irradiated ParRes and SurRes cells were similar when they regrew exponentially. For ParRes all the curves for irradiated and control cells followed each other closely with the same slope and there were only differences in the time for recovery after the irradiations. In SurRes cells the same pattern was observed for all curves except for the 8 Gy curve, which had a lower slope in the exponential phase. The time scale related to regrowth showed that the ParRes cell curves, 2-6 Gy, reached the level $10^{10}$ cells within 32-41 days from the time of irradiation, whilst SurRes cells achieved the same cell number within 40-62 days post-irradiation, thus, growth delays were induced.

The growth curves of ParSen and SurSen cells were quite different to each other. The curves for ParSen 0, 2, 4 and 6 Gy had nearly the same slope in the exponential phase, but the curve for 8 Gy deviated and had a lower slope. The SurSen cells grew, in all cases, slower than the parental ParSen cells. The ParSen growth curves, after 2-8 Gy exposures, all reached the $10^{10}$ level within $36-56$ days, while the SurSen growth curves, after 2-8 Gy, did so within 65-103 days.

There were observable re-irradiation induced changes in cell morphology for both ParSen and SurSen cells. After acute exposure to $2 \mathrm{~Gy}$, both ParSen and SurSen cells were unchanged, but for higher acute doses, 4-8 Gy, there were differences. The SurSen cells that survived higher acute doses tended to swell and become abnormally large, but these large cells did not seem to continue to grow. Instead, SurSen cells that grew after 4-8 Gy acute doses were smaller and often densely packed together. Such acute radiation induced differences were not seen for cultures of ParRes and SurRes cells.

Growth extrapolation assay. The results from the evaluation using the growth extrapolation assay are shown in Table I. There was no detectable difference in radiosensitivity between ParRes and SurRes cells and also no difference between the ParSen and SurSen cells. Thus, there were no signs of radiation induced radioresistance.

Growth delays. The obtained growth delays are shown in Fig. 4. The growth delays were similar for ParRes and SurRes cells after exposures to 2-6 Gy but after 8 Gy there was an increase in the growth delay for the SurRes cells (Fig. 4a). All growth delays, independent of radiation dose, were 

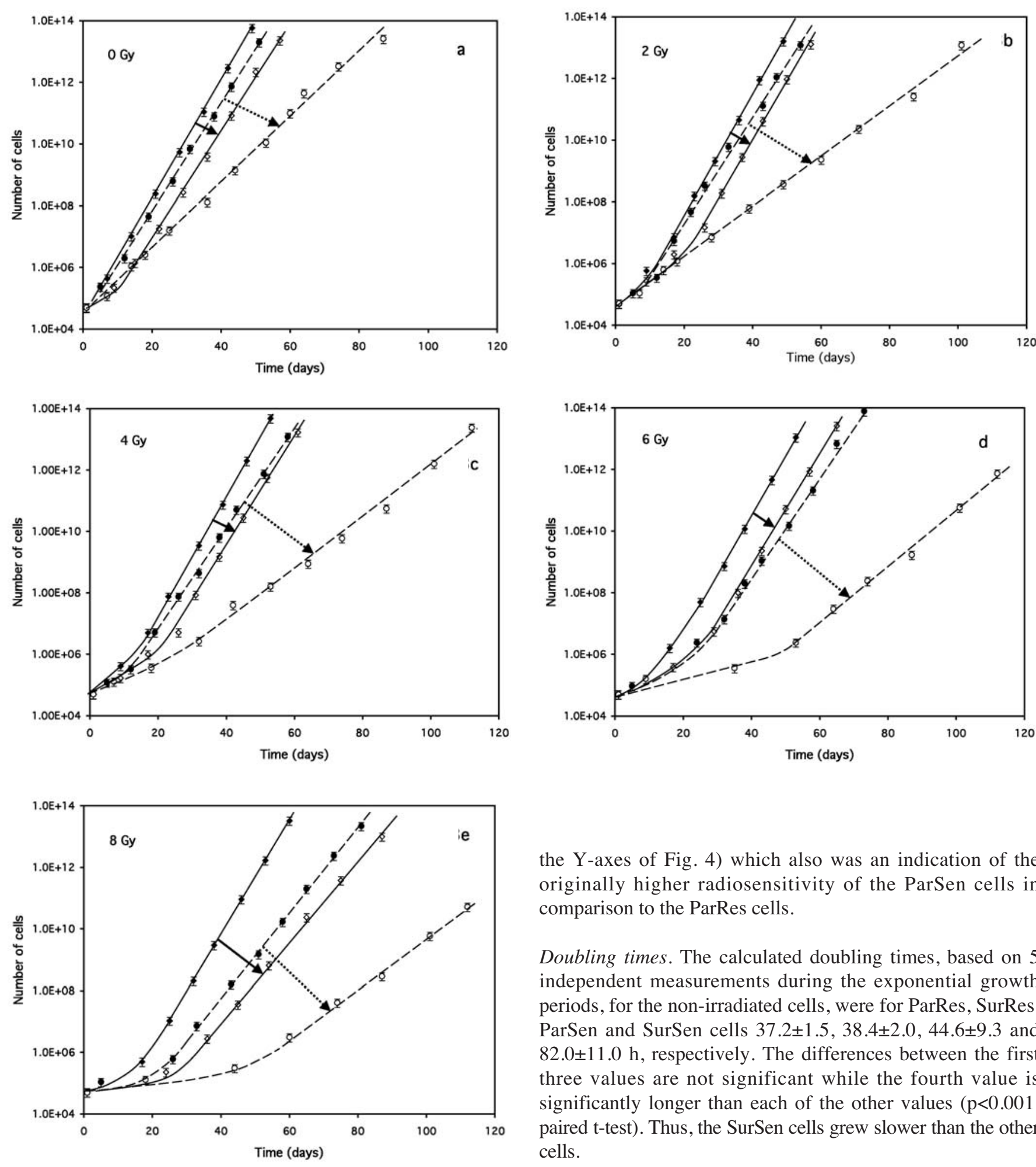

the Y-axes of Fig. 4) which also was an indication of the originally higher radiosensitivity of the ParSen cells in comparison to the ParRes cells.

Doubling times. The calculated doubling times, based on 5 independent measurements during the exponential growth periods, for the non-irradiated cells, were for ParRes, SurRes, ParSen and SurSen cells $37.2 \pm 1.5,38.4 \pm 2.0,44.6 \pm 9.3$ and $82.0 \pm 11.0 \mathrm{~h}$, respectively. The differences between the first three values are not significant while the fourth value is significantly longer than each of the other values $(p<0.001$, paired $t$-test). Thus, the SurSen cells grew slower than the other cells.

Figure 3. Direct comparisons of growth between ParRes (filled diamonds and solid line), SurRes (open diamonds and solid line), ParSen (filled circles and dashed line) and SurSen (open circles and dashed line) cells for various acute radiation doses. The doses were 0 (a), 2 (b), 4 (c), 6 (d) and 8 (e) Gy. The solid arrows indicate, in all panels, the changed growth of SurRes cells in relation to ParRes cells and dotted arrows indicate the changed growth of SurSen in relation to ParSen cells. Mean values and maximal variations from four parallel samples.

longer for the SurSen cells than for the ParSen cells (Fig. 4b). The growth delays for the ParRes cells were only about half of the values for the ParSen cells (note different scaling on

DNA content. The DNA content determination was done using chicken red blood cells as a calibration standard. The DNA content of the chicken cells could be related to the DNA content of a diploid human cell, since they have $35 \%$ of the DNA of a human diploid cell. It was found that the ParRes, SurRes, ParSen and SurSen cells had about 1.27, $1.34,1.58$ and 2.88 times more DNA, respectively, than a diploid human cell. The uncertainty in these determinations was $<5 \%$. Thus, the SurSen cells increased their DNA content with about $82 \%$ in relation to the ParSen cells which was a significant difference $(\mathrm{p}<0.001$, paired t-test). The measured difference between ParRes and SurRes was negligible. 
Table I. Survival as a function of the acute re-irradiation doses.

\begin{tabular}{cllll}
\hline Radiation dose (Gy) & ParRes cells & SurRes cells & ParSen cells & SurSen cells \\
\hline 0 & 1.00 & 1.00 & 1.00 & 1.00 \\
2 & $0.30 \pm 0.09$ & $0.35 \pm 0.11$ & $0.23 \pm 0.07$ & $0.20 \pm 0.06$ \\
4 & $0.100 \pm 0.031$ & $0.086 \pm 0.026$ & $0.058 \pm 0.017$ & $0.053 \pm 0.016$ \\
6 & $0.039 \pm 0.012$ & $0.022 \pm 0.006$ & NA & NA \\
\hline
\end{tabular}

Mean values and maximal variations from four parallel samples. NA, not analysed.
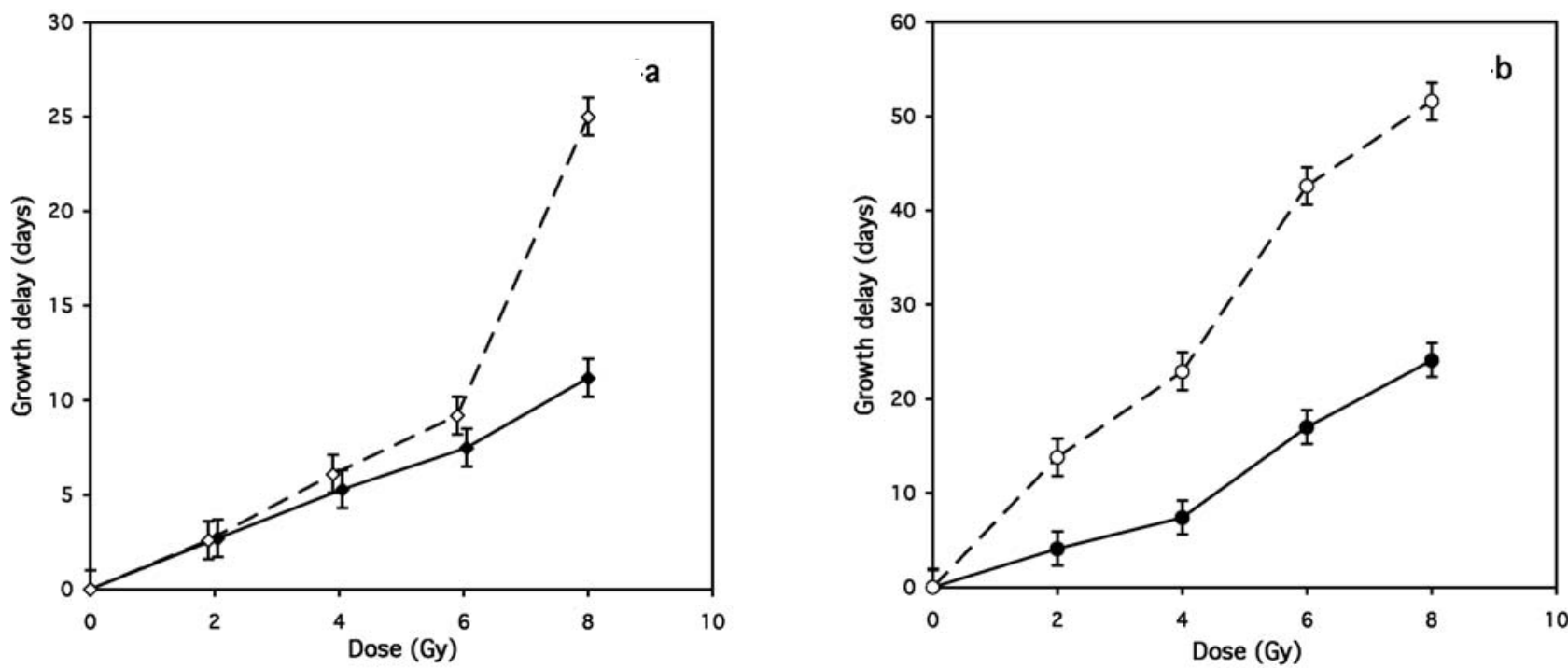

Figure 4. Growth delay versus dose for ParRes cells (filled diamonds and solid line) and SurRes cells (open diamonds and dashed line) (a) and ParSen cells (filled circles and solid line) and SurSen cells (open circles and dashed line) (b). Mean values and maximal variations from four parallel samples.

Cell cycle analysis. The fraction of $\mathrm{G}_{1}$ cells increased (from $36 \pm 3 \%$ to $54 \pm 6 \%$ ) and the fraction of $\mathrm{G}_{2}$ cells decreased (from $46 \pm 1 \%$ to $21 \pm 2 \%$ ) for the SurSen cells in comparison to ParSen cells. A change in the other direction was found for the SurRes cells in comparison to the ParRes cells in that the fraction of $\mathrm{G}_{1}$ cells decreased (from $51 \pm 6 \%$ to $37 \pm 8 \%$ ) and the fraction of $\mathrm{G}_{2}$ cells increased (from $13 \pm 1 \%$ to $35 \pm 2 \%$ ). No noticeable changes were seen in the fraction of cells in S-phase neither for the SurRes nor for the SurSen cells, in comparison to their corresponding parental cells.

Apoptosis. The ParSen cells had apoptotic indices, three days after high dose-rate radiation exposures, in the range $0-2 \%$ while the SurSen cells had values in the range 3-5\%. However, in none of the cases there was a clear radiation dose-effect relation since the controls had approximatively the same levels as the irradiated cells. The apoptotic capacity for ParRes cells, i.e. U118MG cells, has previously been reported to be low (23) and it was not possible to measure apoptotic values with good statistics for either ParRes or SurRes cells.

\section{Discussion}

The growth curves of the control ParRes and SurRes cells (Fig. 3a) were rather similar with the exception that there was a time delay before the SurRes cells started to grow exponentially. This delay was probably required for the reproductive capacity to be restored after the previous high dose, low dose-rate irradiation used for the selection of survivors. For ParRes cells all curves for irradiated and control cells (Fig. 3) followed each other closely with the same slope and only differences in the time for recovery after the irradiations. In SurRes cells the same pattern was observed for all curves except for the dose 8 Gy (Fig. 3e), which had a lower slope and a longer growth delay. Worth noting is that even though the single dose $8 \mathrm{~Gy}$ is rather high, at least compared to the 2 Gy that is normally given per day in external radiotherapy $(15,16)$, both ParRes and SurRes cells were able to regrow and stabilize their reproductive capacity within 60 days after irradiation. A low apoptotic capacity of the ParRes cells, i.e. U118MG cells, has been previously reported (23). Furthermore, there were no significant differences in the amount of DNA and no differences in radiosensitivity between the ParRes and SurRes cells.

For SurSen cells the growth in all cases, controls and irradiated, was much slower than for the parental ParSen cells (Fig. 3). The time before the cells reached exponential growth was delayed with increasing radiation doses for both ParSen and SurSen cells. The curves for ParSen control and the irradiated, 2-6 Gy, had nearly the same slope, but the 
curve for 8 Gy deviated with a lower slope. Furthermore, all growth delays, independent of radiation dose, were longer for the SurSen cells than for the ParSen cells (Fig. 4b). The SurSen cells increased their DNA content and the fraction of $\mathrm{G}_{1}$ cells in comparison to the ParSen cells. The ParSen cells had apoptotic indices in the range 0-2\% while the SurSen cells had values in the range $3-5 \%$. All these changes suggest that the SurSen cells had undergone rather extensive changes as a consequence of the high dose, low dose-rate, beta irradiation that they previously had survived. In spite of these changes we could not detect indications of changes in radiosensitivity when applying the growth extrapolation assay (Table I).

There was an initial and inherent difference in radiosensitivity between the ParRes and the ParSen cells. In a previous study (23) low dose-rate acute effects in U118MG (ParRes) and U373MG (ParSen) cells were reported. In that study, the initial dose-rate was only 0.05-0.09 Gy/h and the exposure time 7 days. As expected, all cultures did regrow after such treatments. It was shown that the U373MG cells had, at day 7 , the strongest cell number reduction and showed radiation induced apoptosis. The U118MG cells had surprisingly low cell number reductions, a $\mathrm{G}_{2}$ block but no radiation induced apoptosis. This is in accordance with the results reported in the present study. Thus, a possible explanation for the initial difference in radiosensitivity might be cell type-dependent differences in the capacity for low dose-rate induced apoptosis.

It seems as if differences in hyperradiosensitivity, HRS, (measured at doses $<0.5$ Gy but at high dose-rate) are not of much importance for these cells, since the U118MG cells have been reported to have HRS, while U373MG cells have not (24). If HRS would have been of importance under the initial low dose-rate exposure conditions, then the U373MG cells should have been more resistant than the U118MG cells. These results are in line with recent data, which indicated that HRS does not influence the outcome of hyperfractionated radiation therapy when human glioma cells were treated as xenografts (30).

It has previously, in a few experimental studies, been indicated that radiation induced changes in radioresponse, e.g. changes in growth rate and radiosensitivity (31) can occur. Genomic instability of tumour cells with high mutation rates in combination with the selective pressure of radiation therapy can be a basis for the development of more resistant sub-clones that contributes to therapy failure. It has been demonstrated that permanently radioresistant cell clones can be induced after irradiation of the human-hamster hybrid GM10115 cells (18). It has also been shown that upregulation of IFN-related signalling pathways, especially upregulation of STAT1, can be a reason for transient radiation induced radioresistance $(32,33)$.

It is necessary to distinguish between mutation induced permanent changes in radiosensitivity and metabolism induced transient changes. A clue to molecular factors involved in transient sensitivity changes came from a report, showing that activation or inhibition of the DNA-damage sensor ATM is of importance (34). It was found that DNA damage inflicted at low rate failed to activate ATM. However, if ATM was activated by chloroquine the cells survived the low dose-rate irradiations better. Thus, ATM activation seems to be an important factor, at least for transient changes in radiosensitivity. The phenomenon called adaptive response, meaning that a low radiation dose can transiently make the exposed cells more resistant to a following high radiation dose, has been shown for some cell types (35). This can be considered as a form of radiation induced radioresistance although it is transient. A similar phenomenon is the recovery from hyperradiosensitivity, HRS, which is called increased radioresistance, IRR $(24,36)$ and that also means transient resistance. However, adaptive response and IRR seems to be of importance only when cells initially are exposed to low radiation doses, $<1 \mathrm{~Gy}$. Thus, IRR is probably of limited interest for radiotherapy of tumours since higher radiation doses are then applied. These phenomena can be of more interest for understanding of radiation effects on surrounding normal tissues, which often are exposed to low doses.

Activation of the epidermal growth factor receptor, EGFR, through interaction with ligands can also induce transient radioresistance $(37,38)$. This activation is assumed to disappear as soon as the ligand mediated stimulation, e.g. by blocking with the antibody cetuximab (39), disappears. Constitutive activation of EGFR, via transfection with EGFRvIII, seems to give permanent radioresistance (40). Radiation can itself transiently activate EGFR (41) but whether radiation can induce mutations so that EGFR is constitutively and permanent active is not known.

The transient and permanent radiation induced changes in growth have mainly been demonstrated in laboratory studies. No clinical data, to the knowledge of the authors, support importance of radiation induced changes in radiosensitivity due to external radiotherapy or due to low dose-rate radionuclide therapy of tumours. If that were the case, many clinical reports would most likely have been published on such a problem (compare with the large number of published reports on treatment induced chemotherapy resistance). However, we expect that this field will be further explored since more information is needed on radiation induced changes in human tumour cells.

\section{Acknowledgments}

The authors acknowledge technical support by Dr Jan Grawé regarding flow cytometry measurements. Financial support from the Swedish Cancer Society, grant 0980-B06-19XBC, and Vinnova, grant 2004-02159, is acknowledged.

\section{References}

1. Witzig TE: Radioimmunotherapy for B-cell non-Hodgkin lymphoma. Best Pract Res Clin Haematol 19: 655-668, 2006.

2. Goldenberg DM and Sharkey RM: Advances in cancer therapy with radiolabeled monoclonal antibodies. Q J Nucl Med Mol Imaging 50: 248-264, 2006.

3. Kwekkeboom DJ, Mueller-Brand J, Paganelli G, Anthony LB, Pauwels S, Kvols LK, O'dorisio TM, Valkema R, Bodei L, Chinol M, Maecke HR and Krenning EP: Overview of results of peptide receptor radionuclide therapy with 3 radiolabeled somatostatin analogs. J Nucl Med 46 (Suppl. 1): S62-S66, 2005.

4. Van Essen M, Krenning EP, De Jong M, Valkema R and Kwekkeboom DJ: Peptide receptor radionuclide therapy with radiolabelled somatostatin analogues in patients with somatostatin receptor positive tumours. Acta Oncol 46: 723-734, 2007. 
5. Pashankar FD, O'Dorisio MS and Menda Y: MIBG and somatostatin receptor analogs in children: current concepts on diagnostic and therapeutic use. J Nucl Med 46: S55-S61, 2005.

6. Carlsson J, Forssell Aronsson E, Hietala SO, Stigbrand T and Tennvall J: Tumour therapy with radionuclides: assessment of progress and problems. Radiother Oncol 66: 107-117, 2003.

7. Larson SM and Krenning EP: A pragmatic perspective on molecular targeted radionuclide therapy. J Nucl Med 46 (Suppl. 1): S1-S3, 2005.

8. DeNardo SJ and DeNardo GL: Targeted radionuclide therapy for solid tumors: an overview. Int J Radiat Oncol Biol Phys 66 (Suppl. 2): S89-S95, 2006.

9. De Jong M, Breeman WAP, Valkema R, Bernard BF and Krenning EP: Combination radio-nuclide therapy using ${ }^{177} \mathrm{Lu}-$ and ${ }^{90}$ Y-labeled somatostatin analogs. J Nucl Med 46: S13-S17, 2005.

10. Carlsson J, Ren ZP, Wester K, Sundberg AL, Heldin NE, Hesselager G, Persson M, Gedda L, Tolmachev V, Lundqvist H, Blomquist $\mathrm{E}$ and Nister M: Planning for intracavitary antiEGFR radionuclide therapy of gliomas. Literature review and data on EGFR expression. J Neurooncol 77: 33-45, 2006.

11. Dillehay LE and Williams JR: Radiobiology of dose-rate patterns achievable in radioimmuno-globulin therapy. Front Radiat Ther Oncol 24: 96-103, 1990.

12. Mutha AD: Radiobiology of low-dose-rate radiation relevant to radioimmunotherapy. Cancer Biother Radiopharm 15: 7-14, 2000.

13. Hernandez MC and Knox SJ: Radiobiology of radioimmunotherapy: targeting CD20 B-cell antigen in non-Hodgkin's lymphoma. Int J Radiat Oncol Biol Phys 59: 1274-1287, 2004.

14. Dale R and Jones B: Radiobiological modelling in radiation oncology. BIR, the British Institute of radiology, London (ISBN 13978-0-905749-60-0), 2007.

15. Steel GG: Basic clinical radiobiology. Arnold Publisher and Hodder Headline Group. London (ISBN 034080783 0), 2002.

16. Hall EJ and Giaccia AJ: Radiobiology for the radiologist. Chapter 5. Lippincott Williams \& Wilkins. Philadelphia (ISBN 0-7817-4151-3), 2006.

17. Bedford JS and Mitchell JB: Dose-rate effects in synchronous mammalian cells in culture. Radiat Res 54: 316-327, 1973.

18. Limoli CL, Corcoran JJ, Jordan R, Morgan WF and Schwartz JL: A role for chromosomal instability in the development of and selection for radioresistant cell variants. Br J Cancer 84: 489-492, 2001.

19. Carlsson J, Eriksson V, Stenerlow B and Lundqvist H: Requirements regarding dose rate and exposure time for killing of tumour cells in beta particle radionuclide therapy. Eur J Nucl Med Mol Imaging 33: 1185-1195, 2006.

20. Essand M, Gronvik C, Hartman T and Carlsson J: Radioimmunotherapy of prostatic adeno-carcinomas: effects of ${ }^{131} \mathrm{I}$ labelled E4 antibodies on cells at different depth in DU 145 spheroids. Int J Cancer 63: 387-394, 1995.

21. O'Donoghue JA, Bardies M and Wheldon TE: Relationships between tumor size and curability for uniformly targeted therapy with beta-emitting radionuclides. J Nucl Med 36: 1902-1909, 1995.

22. Hartman T, Lundqvist H, Westlin JE and Carlsson J: Radiation doses to the cell nucleus in single cells and cells in micrometastases in targeted therapy with ${ }^{131}$ I labelled ligands or antibodies. Int J Radiat Oncol Biol Phys 46: 1025-1036, 2000.

23. Carlsson J, Hakansson E, Eriksson V, Grawe J, Wester K, Grusell E, Montelius A and Lundqvist H: Early effects of low dose-rate radiation on cultured tumor cells. Cancer Biother Radiopharm 18: 663-670, 2003.

24. Joiner MC, Marples B, Lambin P, Short SC and Turesson I: Low-dose hypersensitivity: current status and possible mechanisms. Int J Radiat Oncol Biol Phys 49: 379-389, 2001.

25. Nias AH and Fox M: Minimum clone size for estimating normal reproductive capacity of cultured cells. Br J Radiol 41: 468-474, 1968.
26. Johansson L, Nilsson K, Carlsson J, Larsson B and Jakobsson P Radiation effects on cultured human lymphoid cells. Analysis using the growth extrapolation method. Acta Radiol Oncol 20: $51-59,1981$

27. Sundberg AL, Almqvist Y, Orlova A, Blomquist E, Jensen HJ, Gedda L, Tolmachev V and Carlsson J: Combined effect of gefitinib ('Iressa', ZD1839) and targeted radiotherapy with 211At-EGF. Eur J Nucl Med Mol Imaging 30: 1348-1356, 2003.

28. Mirzaie-Joniani H, Eriksson D, Johansson A, Lofroth PO, Johansson L, Ahlstrom KR and Stigbrand T: Apoptosis in HeLa Hep2 cells is induced by low-dose, low-dose-rate radiation. Radiat Res 158: 634-640, 2002.

29. Mirzaie-Joniani H, Eriksson D, Sheikholvaezin A, Johansson A, Lofroth PO, Johansson L and Stigbrand T: Apoptosis induced by low-dose and low-dose-rate radiation. Cancer 94 (Suppl. 4): $1210-1214,2002$

30. Krause M, Wohlfarth J, Georgi B, Pimentel N, Dorner D, Zips D, Eicheler W, Hessel F, Short SC, Joiner MC and Baumann M: Low-dose hyperradiosensitivity of human glioblastoma cell lines in vitro does not translate into improved outcome of ultrafractionated radiotherapy in vivo. Int J Radiat Biol 81: 751-758, 2005.

31. Suit HD: Response to X-irradiation of a tumour recurring after a TCD95 radiation dose. Nature 211: 996-997, 1966.

32. Khodarev NN, Beckett M, Labay E, Darga T, Roizman B and Weichselbaum RR: STAT1 is overexpressed in tumors selected for radioresistance and confers protection from radiation in transduced sensitive cells. Proc Natl Acad Sci USA 101: 1714-1719, 2004.

33. Fryknäs M, Dhar S, Oberg F, Rickardson L, Rydåker M, Göransson H, Gustafsson M, Pettersson U, Nygren P, Larsson R and Isaksson A: STAT1 signaling is associated with acquired crossresistance to doxorubicin and radiation in myeloma cell lines. Int J Cancer 120: 189-195, 2007.

34. Collis SJ, Schwaninger JM, Ntambi AJ, Keller TW, Nelson WG, Dillehay LE and Deweese TL: Evasion of early cellular response mechanisms following low level radiation-induced DNA damage. J Biol Chem 279: 49624-49632, 2004.

35. Murray D and McEwan AJ: Radiobiology of systemic radiation therapy. Cancer Biother Radiopharm 22: 1-23, 2007.

36. Marples B, Wouters BG, Collis SJ, Chalmers AJ and Joiner MC: Low-dose hyper-radiosensitivity: a consequence of ineffective cell cycle arrest of radiation-damaged G2-phase cells. Radiat Res 161: 247-255, 2004.

37. Liang K, Ang KK, Milas L, Hunter N and Fan Z: The epidermal growth factor receptor mediates radioresistance. Int J Radiat Oncol Biol Phys 57: 246-254, 2003.

38. Toulany M, Kasten-Pisula U, Brammer I, Wang S, Chen J, Dittmann K, Baumann M, Dikomey E and Rodemann HP: Blockage of epidermal growth factor receptor-phosphatidylinositol 3-kinase-AKT signaling increases radiosensitivity of K-RAS mutated human tumor cells in vitro by affecting DNA repair. Clin Cancer Res 12: 4119-4126, 2006.

39. Lennartsson J, Carlsson J and Stenerlöw B: Targeting the epidermal growth factor receptor family in radionuclide therapy of tumors-signal transduction and DNA repair. Lett Drug Design Discov 3: 357-368, 2006.

40. Weppler SA, Li Y, Dubois L, Lieuwes N, Jutten B, Lambin P, Wouters BG and Lammering G: Expression of EGFR variant vIII promotes both radiation resistance and hypoxia tolerance. Radiother Oncol 83: 333-339, 2007.

41. Toulany M, Baumann M and Rodemann HP: Stimulated PI3KAKT signaling mediated through ligand or radiation-induced EGFR depends indirectly, but not directly, on constitutive K-Ras activity. Mol Cancer Res 5: 863-872, 2007. 